finden, der unsinnig genug wäre, zu behaupten, er besitze die positive Idee einer wirklichen unendlichen Zahl." Rund zweihundert Jahre später fand sich der „Unsinnige“. 1883 schrieb Cantor:

Die bisherige Darstellung meiner Untersuchungen ... ist an einen Punkt gelangt, wo ihre Fortführung von einer Erweiterung des ... Zahlbegriffs über die bisherigen Grenzen hinaus abhängig wird, und zwar fällt diese Erweiterung in eine Richtung, in welcher sie meines Wissens bisher von niemandem gesucht worden ist ... Es handelt sich um eine Erweiterung resp. Fortsetzung der Zahlenreihe über das Unendliche hinaus; so gewagt dies auch scheinen möchte, kann ich dennoch nicht nur die Hoffnung, sondern die feste Überzeugung aussprechen, daß diese Erweiterung mit der Zeit als eine durchaus einfache, angemessene, natürliche angesehen werden müsse. Dabei verhehle ich mir keineswegs, daß ich mit diesem Unternehmen in einen gewissen Gegensatz zu weitverbreiteten Anschauungen über das mathematische Unendliche und zu häufig vertretenen Ansichten über das Wesen der Zahlgröße mich stelle.
Cantor hatte sich überaus vorsichtig ausgedrückt. Sein ,gewisser Gegensatz zu weitverbreiteten Anschauungen" war in Wirklichkeit so groß, daß der berühmte Zahlentheoretiker Kronecker ihn ungeschminkt einen „Verderber der Jugend" nannte. Für Hilbert hingegen war Cantors Theorie der transfiniten Zahlen , die bewundernswerteste Blüte mathematischen Geistes und überhaupt eine der höchsten Leistungen rein verstandesmäßiger menschlicher Tätigkeit". Urteile können sehr verschieden ausfallen.

\author{
Adresse des Autors: \\ Prof. Dr. Harro Heuser \\ Universität Karlsruhe \\ Kaiserstraße 12 \\ 76128 Karlsruhe
}

\title{
Das Erkenntnisideal der Mathesis universalis
}

\section{von Hans Poser}

Die aristotelische Naturbetrachtung kannte kaum quantitative Aussagen und schon gar nicht den Gedanken einer einheitlichen, auf die Form mathematischer Ausdrücke gegründeten Theorie; dies sollte der Neuzeit vorbehalten bleiben. Programmatisch hatte Galilei gefordert, zu messen, was meßbar sei, und meßbar zu machen, was noch nicht meßbar ist; aber auch daraus erwächst noch keine geschlossene Theorie. Vielmehr war es Descartes, der dieses so wirkmächtige Ideal, gar in Gestalt einer alle wissenschaftliche Erkenntnis umfassenden Einheitswissenschaft, im Plan einer Mathesis universalis in seiner methodologischen Schrift Regulae ad directionem ingenii (Regeln zur Anleitung der Erkenntniskraft) gegen 1623 entwarf. Zwar wurde das Werk nie vollendet; dennoch trug sein Grundgedanke entscheidend zur Ausbildung der neuzeitlichen mathematisierten Erfahrungswissenschaften bei, denn zum einen war der Entwurf unter Fachgelehrten bekannt; Leibniz beispielsweise hatte sich eine Abschrift besorgt. Zum anderen sollte er für Descartes' Denken richtungweisend werden.

In den Regulae geht es Descartes darum, die so erfolgreiche mathematische (oder geometrische) Methode der Analyse und Synthese einschließlich des Ideals einer axiomatischen Darstellung auf alle Wissenschaften überhaupt auszudehnen, um zu einer völlig neuartigen Einheit aller wissenschaftlichen Erkenntnis in Gestalt der Mathesis universalis zu gelangen. Später, im Anschreiben der Principia, wird er nicht nur die Physik, sondern auch Medizin und Ethik als Äste am Baume der Mathematik bezeichnen. So läßt sich das ganze Werk Descartes' als eine Verfolgung des Programms verstehen, zu dem die Regulae den Grund legten.

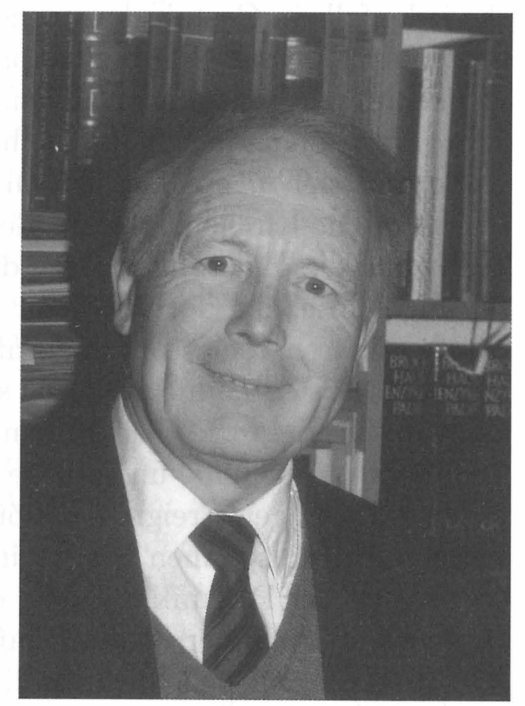

Worum geht es? Die Schrift sollte aus drei Teilen zu je zwölf Regeln „zur Ausrichtung der Erkenntniskraft" bestehen. Sie beabsichtigte, die geometrische Algebra zu einer Universalwissenschaft dergestalt zu erweitern, daß unsere Erkenntniskraft (ingenium) „über alles, was vorkommt, unerschütterliche und wahre Urteile hervorbringt" (R. I), denn „alle Wissenschaft ist zuverlässige und evidente Erkenntnis" (R. II). Das, wodurch die Erkenntniskraft geleitet werden soll, ist die Methode: „Zur wissenschaftlichen Forschung ist Methode notwendig" (R. IV). Sie soll — im Gegensatz zur scholastischen, am jewei- 
ligen Gegenstand ausgerichteten Methodik — nicht disziplinspezifisch, sondern universell sein; eben darum begründet sie als ihr Resultat eine Einheit der Erkenntnis. Instinktiv, meint Desartes, hätten große Talente diese Denkweise früher schon "durchschaut" (R. IV.3); nun aber gelte es, sie von den Anfängen menschlicher Vernunft „bis zur Ermittlung der Wahrheit aus jedem beliebigen Gegenstand zu erweitern" (R. IV.4; alte Zählung: R. IV.5). Hierbei definiert Descartes Methode als „zuverlässige und leicht zu befolgende Regel, so daß, wer sich pünktlich an sie hält, niemals etwas Falsches für wahr unterstellt und, indem er keine geistige Mühe nutzlos verschwendet, sondern sein Wissen Stück für Stück ständig erweitert, die wahre Erkenntnis alles dessen erreicht, wozu er fähig ist." (R. IV.1) Erläuternd fügt er hinzu: „Wenn aber die Methode richtig darüber aufklärt, wie man sich der geistigen Intuition zu bedienen hat [... ] und wie man die Deduktionen ausfindig machen muß, um zu einer umfassenden Erkenntnis zu kommen, so scheint nichts weiter zu ihrer Vollständigkeit erforderlich" (R. IV.2).

Die neue Methode stützt sich also insgesamt auf Regeln. Diese haben nichts mit logischen Schlußregeln zu tun; von Logik ist gar nicht die Rede (oder doch allenfalls implizit, wenn von Deduktionen gesprochen wird), ja, über die traditionelle scholastische Logik spottet Descartes: Einem schon vorhandenen Wissen werde mit ihr ein Schein von Ordnung aufgezwängt. Ihm hingegen geht es um ganz andere Regeln, solche nämlich, die man befolgen muß, um zu gesicherter Erkenntnis gelangen zu können. Doch was sind eigentlich Regeln? Sie sind selbst nicht wahr oder falsch, denn sie beschreiben nichts; sie schreiben vielmehr vor, sie sind mithin präskriptiv oder normativ. Regeln sind überdies etwas ganz Methodisches: Man wird weder von der Natur allgemein noch von einem Tier sagen können, sie folgten einer Regel im Sinne einer Vorschrift. Regeln — das macht ihr Wesen aus — sind hinsichtlich ihrer Verbindlichkeit nicht zu beweisen, sondern nur zu rechtfertigen. Das gilt insbesondere für die Regeln methodischer Erkenntnis, die ja gerade bestimmen, wie man zu Erkenntnissen gelangt; und deshalb können sie nicht ihrerseits den Status einer solchen Erkenntnis haben. Die Rechtfertigung der Regeln besteht denn auch nur partiell in ihrem Erfolg; in erster Linie ergibt sie sich — auch wenn Descartes das alles nicht explizit sagt - aus dem Ziel, dem erkennenden Subjekt eine Methode zur Erkenntnissicherung an die Hand zu geben. Solche Regeln sind die Artikulation einer Methode, die anzunehmen die Vernunft mich angesichts dieses Zieles zwingt.

Wie im einzeln vorzugehen sei, wird in Regel V gesagt: „Die ganze Methode besteht in der Ordnung und Disposition dessen, worauf man sein geistiges Auge richten muß, um irgendeine Wahrheit zu finden. Und zwar werden wir diese Regel genau befolgen, wenn wir verwickelte und dunkle Propositionen stufenweise auf einfachere zurückführen und sodann von der Intuition der allereinfachsten zur Erkenntnis aller anderen über dieselben Stufen hinaufzusteigen versuchen." Es handelt sich also um nichts anderes als das aus der Geometrie über Jahrhunderte vertraute Verfahren von (Problem-) Analyse mit anschließender Synthese, die zu einem Beweis führt.

\section{Wenn wir also die Sache recht betrachtet haben, so besitzen wir nicht Sicheres in unserem Wissen als unsere Mathematik}

Nikolaus von Kues um 1450

Der Weg zur gesuchten unerschütterlichen evidenten Erkenntnis soll nun durch eine Unterteilung der Fragen in „einfache Propositionen“ und „verwickelte und dunkle Propositionen" bewältigt werden; letztere wiederum werden in "vollkommen verstandene“ und „unvollkommen verstandene Probleme“ zerlegt (R. XIII). So gelten die Regeln I - XII den einfachen Propositionen, die Regeln XII - XXIV sollten dagegen die vollkommen verstandenen Probleme behandeln (fertiggestellt wurde der Text nur bis Regel XVIII, gefolgt von einer Formulierung der bloßen Regeln ohne Erläuterung bis zu Regel XXI). Der wohl nie in Angriff genommene dritte Teil hätte den unverstandenen Problemen gelten sollen, also empirischen Fragen, weil sich diese aufgrund von Daten und Experimenten nie vollkommen beantworten lassen. Durch den Kunstgriff, diese als Hypothesen zu formulieren und damit von Bedingungen abhängig zu machen, sollen die unvollkommen verstandenen Probleme auf vollkommen verstandene zurückgeführt werden $(R$. XIII.1). Wenn aber die vollständig verstandenen Probleme die der Mathematik sind, so ist mit diesem Ansatz ein Reduktionsprogramm empirischer Aussagen auf mathematische Aussagen formuliert.

Alle menschliche Erkenntnis ist entweder Erfahrung oder Mathematik.

Friedrich Nietzsche um 1880

Ein ähnlicher Rückführungsgedanke beherrscht den zweiten Teil. Es geht dort um die Übertragung des damals noch recht neuen Kunstgriffs der Mathematik, in einer Gleichung das Unbekannte mit $x$ zu bezeichnen und wie die schon bekannten Werte zu behandeln, um, vereinfacht gesagt, die Gleichung nach 
$x$ aufzulösen. Direkt lösbare Fragen oder Propositionen sind nun solche, die allein mit den arithmetischen Grundoperationen gelöst werden können (vgl. R. XVII). Sie verlangen an Erkenntniskraft nur, was uns von Natur mitgegeben ist. Die Hauptaufgabe besteht also darin, die Gleichungsauflösung so weit $\mathrm{zu}$ treiben, daß wir eine vollkommen verstandene Frage in eine direkt lösbare überführt haben. Zugleich mit dem Rückführungsprogramm wird der Anspruch Descartes' deutlich, alle überhaupt absicherbare Erkenntnis auf diese Weise erfaßt zu haben, denn wo nachweislich eine Rückführung der skizzierten Art nicht möglich ist, sind wir an unüberschreitbare Grenzen der menschlichen Erkenntniskraft gestoßen (R. VIII)! Wo wir hingegen erfolgreich sind, gelangen wir zu notwendiger und evidenter Erkenntnis. Da die Methode universell ist, konstituiert sie zugleich eine Einheit des Wißbaren, eben eine Universalwissenschaft.

Das bisher Gesagte mag den Eindruck erwecken, wir hätten es in den Regulae mit einem Mathematikbuch zu tun. Doch weit gefehlt, denn es handelt nicht von mathematischen Gleichungen, sondern von der $\mathrm{Me}$ thode deren Lösung. Eben diese Methode soll auf alle Gegenstände der Erkenntnis Anwendung finden. Dabei ist Descartes der Überzeugung, daß wir in der Geometrie oder in der Mathematik, anders als in der Syllogistik, neue Erkenntnisse zu gewinnen vermögen. Der Syllogismus

\section{Alle $A$ sind $B$ \\ Einige $A$ sind $C$ \\ Einige $B$ sind $C$}

bietet uns keinerlei neue Erkenntnisse. Doch wenn wir begriffen haben, daß die Quadratwurzel von 2 geometrisch gewonnen werden kann, nämlich als Diagonale eines Quadrates, dann führt uns der Satz des Pythagoras auch zu einer Konstruktion der Quadratwurzel aus 3, wenn wir auf der Diagonalen des Quadrates der Seitenlänge 1 das Lot errichten und eine Einheitslänge abtragen: Die Hypothenuse des so konstruierten Dreiecks hat die Länge der Quadratwurzel aus 3. Dieses Verfahren läßt sich nun beliebig fortführen und erlaubt uns, geometrisch alle Quadratwurzeln zu konstruieren: Wir gewinnen stets etwas Neues durch Anwendung der (Konstruktions-) Methode! Nun ist dies weder ein Beispiel, das erst Descartes gefunden hätte (schon Platon bedient sich seiner), noch eines aus der Arithmetik; dennoch zeigt es, wieso er glaubte, Geometrie und Mathematik führten zu Neuem, wenn man deren Verfahren methodisch einsetzt. Das aber muß bei der Übertragung der Methode auf alle anderen Gebiete menschlichen Denkens ebenso gelingen!

Auch wenn Descartes all dies in den Regulae nur als Programm formulierte, war damit der Gedanke geboren, wahrhaft gesicherte Erkenntnis nur mit dieser der Mathematik entlehnten Methode gewinnen zu können: Die Mathesis universalis sollte so über Leibnizens Scientia generalis bis zur Suche nach der Einheitswissenschaft im Wiener Kreis zum Leitgedanken der Neuzeit werden und in ihrer Anwendbarkeit die Grundlage unserer technisch-wissenschaftlichen Welt der Gegenwart bilden, bis sich mit Tarski und Gödel unüberschreitbare formale Grenzen aufweisen ließen. Daß uns Zauberlehrlingen diese selbstgeschaffene technisierte Welt heute gravierende Schwierigkeiten bereitet, ist aber trotz aller postmodernen Vorwürfe nicht Descartes anzulasten, denn wir sind es gewesen, die im erfolgreichen Aufbau solcher mathematisierten Wissenschaft die Ethik als Zweig des cartesischen Baumes der Erkenntnis vernachlässigt haben. Daß sie sich wohl einer Mathematisierung entzieht, mag dafür ein Grund, aber keine Entschuldigung sein.
Adresse des Autors:
Prof. Dr. Hans Poser
Institut für Philosophie
Technische Universität
Ernst-Reuter-Platz 7
10587 Berlin

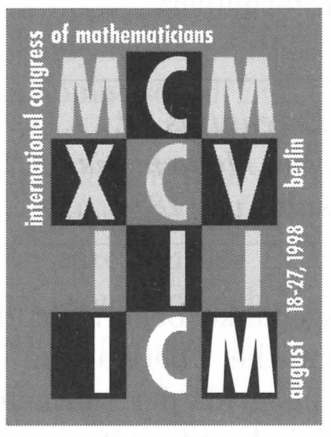

\section{Informationen zum ICM'98 erhält man unter}

http://elib.zib.de/ICM98

Dort findet man alles Wissenswerte zu Organisation, Programm, Satellitenkonferenzen, Internationaler Mathematischer Union (IMU), Fields-Medaillen und anderes mehr. Für Anfragen wende man sich elektronisch an icm98@zib.de oder schriftlich an

ICM'98 c/o Prof. Dr. J. Winkler

TU Berlin, MA 8-2

Straße des 17. Juni 135

D-10623 Berlin, Germany

FAX: +49-30-314 21604 